\title{
Prognostic value of vascular endothelial growth factor-C and podoplanin mRNA expression in esophageal cancer
}

\author{
AGNIESZKA JUCHNIEWICZ ${ }^{1}$, WIESŁAWA NIKLIŃSKA ${ }^{2}$, OKSANA KOWALCZUK ${ }^{1}$, WOJCIECH LAUDAŃSKI ${ }^{3}$, \\ ANETTA SULEWSKA ${ }^{1}$, PIOTR DZIEGIELEWSKI ${ }^{3}$, ROBERT MILEWSKI ${ }^{4}$, WOJCIECH NAUMNIK ${ }^{5}$, \\ MIROSŁAW KOZŁOWSKI ${ }^{3}$ and JACEK NIKLIŃSKI ${ }^{1}$
}

\begin{abstract}
Departments of ${ }^{1}$ Clinical Molecular Biology, ${ }^{2}$ Histology and Embryology, and ${ }^{3}$ Thoracic Surgery, Medical University of Bialystok, 15-267 Białystok; ${ }^{4}$ Department of Statistics and Medical Informatics, Medical University of Bialystok, 15-295 Białystok; ${ }^{5}$ Department of Lung Diseases and Tuberculosis, Medical University of Bialystok, 15-540 Białystok, Poland
\end{abstract}

Received September 3, 2014; Accepted May 29, 2015

DOI: $10.3892 / \mathrm{ol} .2015 .3824$

\begin{abstract}
Vascular endothelial growth factor-C (VEGF-C), VEGF-D, VEGF receptor-3 (VEGFR-3) and podoplanin (PDPN) are involved in the spread of cancer. The current study evaluated VEGF-C, VEGF-D, VEGFR-3 and PDPN mRNA expression levels in 84 esophageal cancer samples from patients who had undergone surgery according to reverse transcription-quantitative polymerase chain reaction, and correlated the results with the clinicopathological features. The effects on lymph node metastasis and survival were identified by performing univariate and multivariate analyses. VEGF-C, PDPN, VEGF-D and VEGFR-3 were overexpressed in 52.4, $52.4,32.1$ and $51.2 \%$ of esophageal cancer samples, respectively. Furthermore, the expression of VEGF-C and PDPN was significantly correlated with lymph node metastasis, depth of tumor invasion and tumor stage $(\mathrm{P}<0.05)$. Logistic regression analysis identified tumor size $(\mathrm{P}=0.001)$, depth of invasion $(\mathrm{P}=0.002)$ and PDPN mRNA expression $(\mathrm{P}=0.022)$ as significant multivariable predictors of regional lymph node metastasis. Upon univariate survival analysis, the depth of tumor invasion, lymph node metastasis, histological grade, tumor stage, tumor size, residual tumor, and VEGF-C and PDPN mRNA expression were identified to be significant independent prognostic factors for overall survival (OS) time. Additionally, multivariate analysis identified tumor size $(\mathrm{P}=0.049)$, residual tumor $(\mathrm{P}<0.001)$ and $\mathrm{PDPN}$ mRNA expression $(\mathrm{P}=0.02)$ as independent factors for poor $\mathrm{OS}$ time. Thus, it was concluded that PDPN mRNA expression may serve as predictor for regional lymph node metastasis, and that VEGF-C and PDPN may be prognostic factors in patients with resected esophageal cancer.
\end{abstract}

Correspondence to: Professor Jacek Niklinski, Department of Clinical Molecular Biology, Medical University of Bialystok, 13 Waszyngtona Street, 15-267 Bialystok, Poland

E-mail: niklinsj@umb.edu.pl

Key words: esophageal cancer, vascular endothelial growth factor-C mRNA, podoplanin mRNA, lymph node metastasis, prognosis

\section{Introduction}

Esophageal carcinoma is the eighth most frequently diagnosed cancer and the sixth leading cause of cancer mortality worldwide, with an estimated 482,000 new cases and 407,000 mortalities in 2008. Patients with esophageal carcinoma have a poorer prognosis in comparison to patients exhibiting any other type of gastrointestinal tumor (1). Lymph node involvement is an important prognostic factor for survival in patients with esophageal carcinoma (2). Despite significant improvements in the diagnosis and available therapeutic strategies for the disease, survival rates remain low. For example, the 5-year survival rate of patients exhibiting esophageal carcinoma with lymph node metastasis who have undergone an esophagectomy and three-field lymphadenectomy is only $15-39 \%$ (3).

Five members of the vascular endothelial growth factor (VEGF) family (VEGF-A, VEGF-C, VEGF-D, VEGF-E and placental growth factor) and their receptors [VEGF receptor (VEGFR)-1, VEGF-2 and VEGF-3] are important in the formation of the vascular network (4). VEGF-C and VEGF-D have been characterized as lymphangiogenic and angiogenic growth factors, and have been demonstrated to signal through the receptors VEGFR-2 and VEGFR-3 in various physiological and pathological processes (5). A mouse model study demonstrated that lymphatic spread and lymphangiogenesis are associated with the expression of VEGF-D or VEGF-C by the tumor cells (6). Furthermore, VEGF-C and VEGF-D appear to be involved in the origin and/or progression of lymphangiogenesis in various different types of cancer, including gastric and esophageal cancer, with overexpression correlated with nodal metastasis and patient survival $(7,8)$.

Podoplanin (PDPN) is 43-kDa mucin-type transmembrane glycoprotein that is expressed in lymphatic endothelial cells, but not in blood endothelial cells (9). PDPN has previously been used to assess lymphatic vessel density and invasion in various types of cancer, including esophageal carcinoma $(10,11)$. Thus, it may act as a mediator of tumor cell invasion and metastasis (12).

The present study evaluated the association between VEGF-C VEGF-D, VEGFR-3 and PDPN mRNA expression 
levels, and the clinicopathological factors and survival of patients with esophageal carcinoma.

\section{Materials and methods}

Patients and tissues. Tumor specimens were obtained from 84 patients with primary esophageal cancer who underwent an esophagectomy at the Department of Thoracic Surgery, Medical University of Białystok (Białystok, Poland). No patients had received pre-operative chemotherapy or radiotherapy. The study population consisted of 76 men $(90.5 \%)$ and 8 women (9.5\%), and the mean age at the time of diagnosis was 63 years (range, 42-82 years). Pathological stage was determined using the seventh edition of the American Joint Committee on Cancer tumor-node-metastasis classification system (13). Following surgery, all patients underwent clinical follow-up evaluations every 3-6 months, including a clinical history, physical examination, laboratory analysis, fiberoptic esophagoscopy, ultrasound examination of the neck and abdomen, barium esophagram, computed tomography (CT) scan, endoscopic ultrasound, positron emission tomography-CT scan, and endobronchial ultrasound if necessary. The mean follow-up time was 25 months (range, 3-101 months). Survival analysis was performed at the termination of follow-up, including an overall survival (OS) analysis. Non-malignant esophageal tissue samples were collected from the same patients at a distance of $3-5 \mathrm{~cm}$ from the tumor (3-8 samples, per patient).

The present study was conducted in accordance with the Declaration of Helsinki, the study protocol was approved by the local Ethics Committee (approval no. R-1-002/28/2010) and written informed consent was obtained from all participants prior to analysis.

RNA extraction and complementary (c)DNA synthesis. Tissue samples were collected intraoperatively. Following macroscopic visual assessment, the samples of tumor tissue and non-malignant esophageal tissue were frozen in liquid nitrogen and stored at $-80^{\circ} \mathrm{C}$. Sections $(4 \mu \mathrm{m})$ of frozen tissue specimens were cut and stained with hematoxylin and eosin (Cryotome $^{\mathrm{TM}}$ FSE cryostat; Thermo Fisher Scientific, Inc., Hemel Hempstead, UK). The presence of carcinoma cells was confirmed by experienced pathologists. Only tumor samples composed of $\geq 50 \%$ tumor cells upon microscopic analysis were used for subsequent processing.

Total RNA was isolated and purified from the tissue specimens using a mirVana ${ }^{\mathrm{TM}}$ miRNA Isolation kit (Ambion Life Technologies, Austin, TX, USA), according to the manufacturer's instructions. The resulting RNA extracts were stored at $-80^{\circ} \mathrm{C}$ until required. RNA quantity was assessed using a NanoDrop 2000c Spectrophotometer (Thermo Fisher Scientific, Inc., Wilmington, DE, USA). RNA quality, including 28S/18S ratio and RNA integrity number, was measured using the 2100 Bioanalyzer (serial no. DE72905449) and an RNA 6000 Nano Assay kit (Agilent Technologies Inc., Santa Clara, CA, USA), according the manufacturer's instructions. Total RNA $(1 \mu \mathrm{g})$ was transcribed into cDNA using High Capacity RNA-to-cDNA Master Mix with No-RT Control (Applied Biosystems Life Technologies, Foster City, CA, USA) in a Labcycler (model no. 1120240193; Sensoquest GmbH, Göttingen, Germany), according to the manufacturers' instructions.
Determining mRNA expression levels. The mRNA expression levels of VEGF-C, VEGF-D, VEGFR-3 and PDPN were evaluated in the tumor and paired non-malignant esophageal tissues by performing comparative reverse transcription-quantitative polymerase chain reaction (RT-qPCR) using commercially available TaqMan ${ }^{\circledR}$ Gene Expression assays (Applied Biosystems Life Technologies) (Table I). Amplification was performed in a 20- $\mu 1$ reaction mixture containing $10 \mu 1$ TaqMan Gene Expression Master Mix (Applied Biosystems Life Technologies), $1 \mu 1$ appropriate TaqMan Gene Expression assay solution and $2 \mu 1$ cDNA solution. The PCR cycle conditions were as follows: $50^{\circ} \mathrm{C}$ for $2 \mathrm{~min}$ and a hold at $95^{\circ} \mathrm{C}$ for $10 \mathrm{~min}$, followed by 40 cycles of $95^{\circ} \mathrm{C}$ at $15 \mathrm{sec}$ and $60^{\circ} \mathrm{C}$ for $1 \mathrm{~min}$. Each sample was analyzed in triplicate. The reaction was conducted on an ABI PRISM ${ }^{\circledR}$ 7900HT Sequence Detection System (SDS; Applied Biosystems Life Technologies) equipped with SDS software (version 2.4) for performing baseline and cycle threshold $(\mathrm{Ct})$ calculations. Gene transcript expression levels were quantified as $\mathrm{Ct}$ values normalized to a reference control gene (18S rRNA), using the following equation: $\Delta \mathrm{Ct}=\mathrm{Ct}_{\mathrm{gene}}-\mathrm{Ct}_{\mathrm{ref}}$. Gene expression levels were inversely proportional to the $\Delta \mathrm{Ct}$ values and were based on a $\log _{2}$ scale. The reaction mixture and cycle conditions for $18 \mathrm{~S}$ rRNA cDNA amplification were the same as those described for VEGF-C, VEGF-D, VEGFR-3 and PDPN cDNA amplification.

Tumor-associated fold-change (FC) in mRNA expression level was calculated using the following equation: $\mathrm{FC}=2^{-\Delta \Delta \mathrm{Ct}}$, where $\Delta \mathrm{Ct}$ equals the difference between the normalized expression of the gene in the tumor samples $\left(\mathrm{Ct}_{\text {gene } \mathrm{T}}\right)$ and its normalized expression in the corresponding non-malignant esophageal tissue $\left(\mathrm{Ct}_{\text {gene } \mathrm{N}}\right)$ (14). Logarithmically transformed $\mathrm{FC}$ values $\left[\log _{2}(\mathrm{FC})\right]$ were used for statistical analysis. A $\log _{2}(\mathrm{FC})$ value of 1.0 was used as the threshold to categorize samples into low $\left[\log _{2}(\mathrm{FC})<1.0\right]$ and high $\left[\log _{2}(\mathrm{FC})>1.0\right]$ gene expression groups.

Statistical analysis. Due to asymmetrical data distribution (as determined by Shapiro-Wilk tests), non-parametric tests were used for all statistical analyses. Categorical data were compared using the $\chi^{2}$ or Fisher's exact probability test. Logistic regression analysis was performed to identify univariable predictors of lymph node metastasis. Significant univariable predictors (and those that were clinically appropriate for inclusion in a model to predict lymph node involvement) were considered in a stepwise logistic regression model. OS times were calculated from the date of surgery to the date of mortality or the most recent follow-up. The Kaplan-Meier method was applied to estimate the probability of survival as a function of time. Differences in the survival of the subgroups of patients were compared using the log-rank test. In addition, the prognostic value of lymphatic vessel invasion was examined by performing univariate and multivariate Cox's proportional hazard models. Statistical analyses were performed using the Statistica (version 10.0; StatSoft Inc., Tulsa, OK, USA) and Stata/IC (version 12.1; StataCorp LP, College Station, TX, USA) software. $\mathrm{P}<0.05$ was considered to indicate a statistically significant difference.

\section{Results}

In the cancerous tissues, a high level of mRNA expression for VEGF-C was observed in 44 patients $(52.4 \%)$, for PDPN in 
Table I. Assays analyzed in the present study.

\begin{tabular}{llrr}
\hline Gene symbol & \multicolumn{1}{c}{ Official gene product name } & Gene ID $^{\mathrm{a}}$ & Assay ID $^{\mathrm{b}}$ \\
\hline VEGF-C & Vascular endothelial growth factor C & 12682 & HS01099203_m1 \\
VEGF-D & Vascular endothelial growth factor D & 3708 & Hs01047677_m1 \\
PDPN & Podoplanin & 29602 & Hs00366766_m1 \\
VEGFR-3 & Vascular endothelial growth factor receptor 3 & 3767 & Hs01128659_m1
\end{tabular}

According to the ${ }^{\mathrm{a}} \mathrm{HUGO}$ gene nomenclature committee and ${ }^{\mathrm{b}}$ Applied Biosystems Life Technologies. VEGF, vascular endothelial growth factor; VEGFR, VEGF receptor; PDPN, podoplanin.

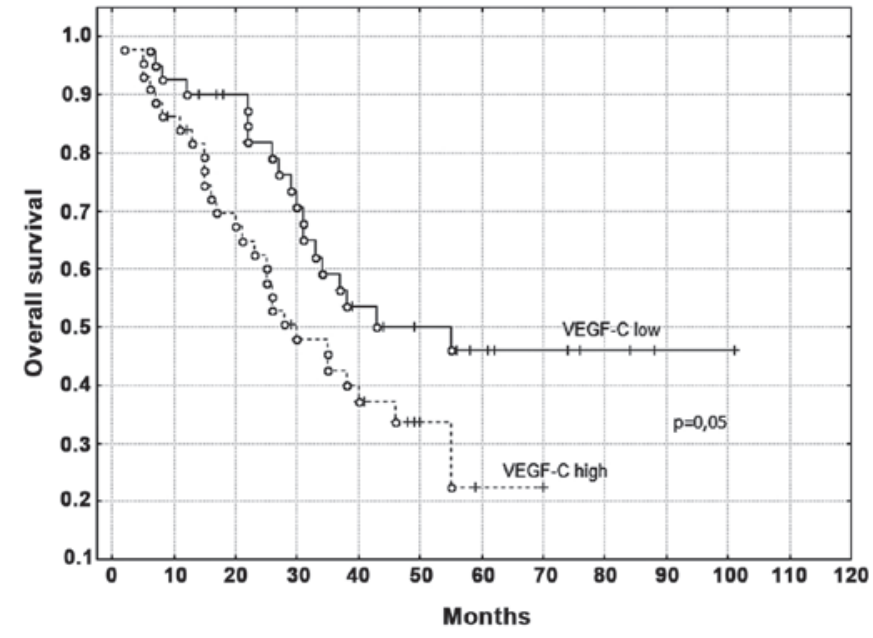

Figure 1. Kaplan-Meier analysis of overall survival according to VEGF-C mRNA expression in patients with esophageal cancer. VEGF-C, vascular endothelial growth factor-C.

44 patients $(52.4 \%)$, for VEGFR-3 in 43 patients $(51.2 \%)$ and for VEGF-D in 27 patients $(32.1 \%)$. The expression of PDPN was significantly correlated with the histological type, tumor stage, lymph node metastasis, depth of tumor invasion and tumor location $(\mathrm{P}<0.05)$. However, there was no significant association between PDPN mRNA expression and age, gender, tumor size, histological grade or residual tumor $(\mathrm{P}>0.05)$. VEGF-C overexpression was significantly associated with tumor depth, tumor stage and lymph node metastasis $(\mathrm{P}<0.05)$. Furthermore, the expression of VEGF-D was significantly associated with histological grade, tumor stage and lymph node metastasis, and VEGFR-3 expression was significantly correlated with tumor size $(\mathrm{P}<0.05)$ (Table II).

To investigate the risk factors associated with lymph node metastasis, univariate and multivariate regression analyses of gender, tumor location, tumor size, depth of invasion, and PDPN, VEGFR-3, VEGF-C and VEGF-D mRNA expression were conducted. Logistic univariate analysis identified that tumor size, depth of invasion, and VEGF-C, VEGF-D and PDPN mRNA expression were all significantly associated with lymph node metastasis $(\mathrm{P}<0.05)$. Among these factors, PDPN mRNA expression $(\mathrm{P}=0.022)$, increasing tumor size $(\mathrm{P}=0.001)$ and increasing depth of invasion $(\mathrm{P}=0.002)$ were significant independent risk factors for lymph node metastasis. The other factors were not predictive for lymph node metastasis (Table III).

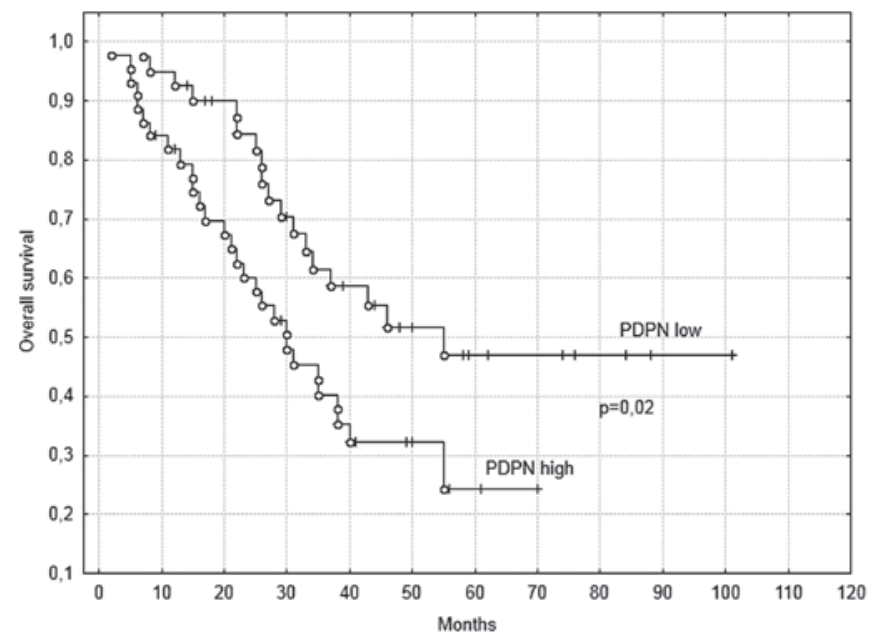

Figure 2. Kaplan-Meier analysis of overall survival according to PDPN mRNA expression in patients with esophageal cancer. PDPN, podoplanin.

The median patient follow-up period was 31 months (range, 2-101 months). For VEGF-C mRNA expression, the median OS time of the patients was 37 months in the low expression group [95\% confidence interval (CI), 29-44 months] and 27 months in the high expression group (95\% CI, 20-38 months; Fig. 1). For VEGF-D mRNA expression, the median OS time of the patients was 31 months in the low expression group (95\% CI, 26-38 months) and 30 months in the high expression group (95\% CI, 14-50). For PDPN mRNA expression, the median OS time was 37 months in the low expression group (95\% CI, 27-46 months) and 28.5 months in the high expression group (95\% CI, 20-38 months) (Fig. 2). Furthermore, for VEGFR-3 mRNA expression, the median OS time of the patients was 34 months in the low expression group (95\% CI, 26-40 months) and 29 months in the high expression group (95\% CI, 21-41 months).

The patients in the high VEGF-C expression group were associated with a significantly shorter OS time following surgery compared with the patients in the low expression group $(\mathrm{P}=0.05$; Fig. 1). Furthermore, the OS time of the patients in the high PDPN expression group was significantly shorter than that in the low expression level ( $\mathrm{P}=0.02$; Fig. 2). By contrast, no association was identified between VEGFR-3 and VEGF-D expression levels and OS time.

In univariate analysis, the following parameters significantly affected OS: Tumor size, depth of tumor invasion, lymph node metastasis, histological grade, tumor stage, 


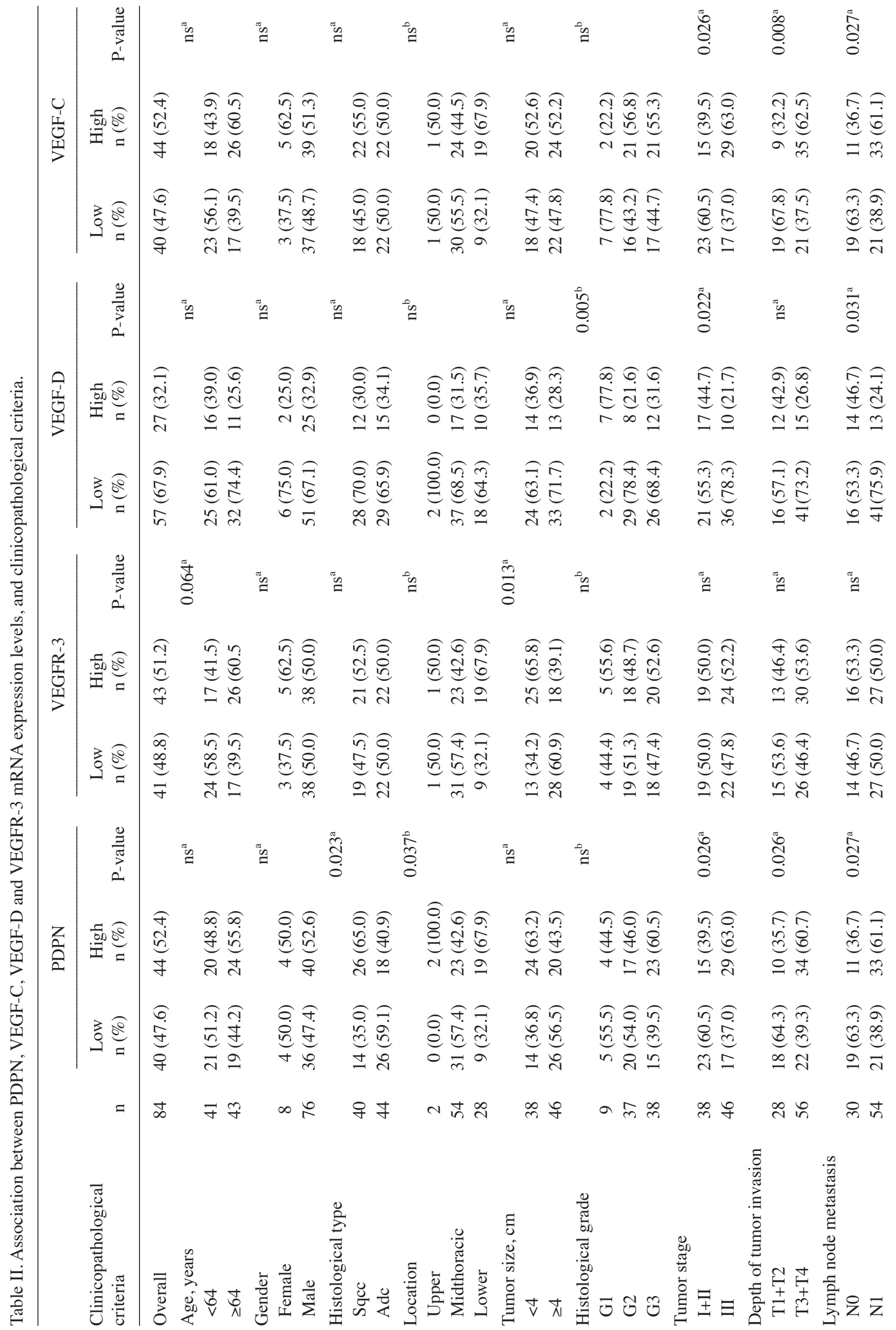


residual tumor, and VEGF-C and PDPN mRNA expression. In multivariate analysis, tumor size, residual tumor and PDPN mRNA expression were identified as independent prognostic factors for a poor OS time in esophageal cancer (Table IV).

\section{Discussion}

Metastasis is directly or indirectly responsible for $>90 \%$ of all cancer mortalities (15). In numerous types of carcinoma, the presence of tumor cells in the lymph nodes is the initial manifestation of metastasis and one of the most important factors of a poor prognosis. The rapid growth and invasive character of esophageal tumors has often been associated with the lymphatic spread of the disease at diagnosis (3).

The induction of lymphangiogenesis by tumors is mediated by growth factors. The most widely investigated factors are members of the VEGF family (VEGF-C, VEGF-D and VEGF-A) and their receptors $(16,17)$. Furthermore, previous studies have indicated that PDPN may be associated with lymphatic dissemination and prognosis in patients with esophageal cancer (18).

The aim of the present study was to analyze the association between the mRNA expression of VEGF-C, VEGF-D, VEGFR-3 and PDPN, and the clinicopathological factors and outcomes of patients with esophageal cancer. Overexpression of VEGF-C, VEGF-D, VEGFR-3 and PDPN was observed in the cancerous tissue samples. These findings are in accordance with the results of studies by Kimura et al (19) and Okazawa et al (20), and our previous study (8), which used immunohistochemistry to demonstrate that esophageal tumors express VEGF-C and VEGF-D. In addition, Tanaka et al (21) used RT-qPCR to demonstrate that esophageal cancer cells express VEGF-C, and Tong et al (22) and Rahadiani et al (23) identified PDPN overexpression in esophageal carcinoma.

Previous studies have demonstrated that increased VEGF-C and VEGF-D expression is correlated with increased tumor cell dissemination to the regional lymph nodes in a range of primary human carcinomas, including esophageal cancer $(8,20,21)$. The present study identified that VEGF-D mRNA overexpression was associated with histological grade, tumor stage and lymph node metastasis. The study by Tzao et al (24) and our previous study (8) obtained similar results. In the current study, a high expression level of VEGF-C was significantly correlated with tumor stage, depth of tumor invasion and lymph node metastasis. This is in accordance with previous studies by Okazawa et al (20), Tanaka et al (21) and Kitadai et al (25), which identified a close correlation between VEGF-C expression and depth of tumor invasion, tumor stage and lymph node metastasis. In the patients with esophageal cancer, VEGFR-3 expression was only correlated with tumor size.

The current study identified that PDPN overexpression was correlated with tumor stage, depth of tumor invasion, lymph node metastasis, tumor location and histological type. The current findings are in agreement with those obtained by Nakayama et al (18), Rahadiani et al (23) and Tong et al (22), which demonstrated a significant correlation between PDPN tumor expression and pathological stage, depth of tumor invasion and lymph node metastasis in esophageal carcinoma.

Furthermore, the present study demonstrated that VEGF-C, VEGF-D and PDPN mRNA expression, tumor 
Table III. Univariate and multivariate analysis of risk factors for lymph node metastases.

\begin{tabular}{|c|c|c|c|c|c|c|}
\hline \multirow[b]{2}{*}{ Factor } & \multicolumn{3}{|c|}{ Univariate analysis } & \multicolumn{3}{|c|}{ Multivariate analysis } \\
\hline & Odds ratio & $95 \% \mathrm{CI}$ & P-value & Odds ratio & $95 \%$ CI & P-value \\
\hline Gender & 3.400 & $0.752-15.379$ & ns & & & ns \\
\hline Tumor location & 0.712 & $0.297-1.706$ & ns & & & ns \\
\hline Tumor size & 15.667 & $5.144-47.713$ & $<0.001$ & 8.286 & $2.355-29.158$ & 0.001 \\
\hline Depth of tumor invasion & 6.531 & $2.408-17.718$ & $<0.001$ & 10.272 & $2.277-46.331$ & 0.002 \\
\hline VEGFR-3 mRNA expression & 0.875 & $0.358-2.139$ & ns & & & ns \\
\hline VEGF-C mRNA expression & 2.714 & $1.079-6.827$ & 0.034 & & & ns \\
\hline VEGF-D mRNA expression & 0.362 & $0.140-0.939$ & 0.036 & 0.315 & $0.084-1.184$ & ns \\
\hline PDPN mRNA expression & 2.714 & $1.079-6.827$ & 0.034 & 5.980 & $1.301-27.481$ & 0.022 \\
\hline
\end{tabular}

CI, confidence interval; VEGF, vascular endothelial growth factor; VEGFR, VEGF receptor; PDPN, podoplanin; ns, not significant (P>0.05).

Table IV. Cox regression analysis of independent factors affecting overall survival.

\begin{tabular}{|c|c|c|c|c|c|c|}
\hline \multirow[b]{2}{*}{ Factor } & \multicolumn{3}{|c|}{ Univariate } & \multicolumn{3}{|c|}{ Multivariate } \\
\hline & HR & $95 \% \mathrm{CI}$ & $\mathrm{P}$-value & HR & $95 \% \mathrm{CI}$ & P-value \\
\hline Depth of tumor invasion & 3.765 & $1.802-7.87$ & $<0.001$ & 2.191 & $0.957-5.014$ & ns \\
\hline Lymph node metastasis & 7.72 & $3.099-19.231$ & $<0.001$ & & & ns \\
\hline Histological grade & 1.832 & $1.156-2.905$ & 0.010 & & & ns \\
\hline Tumor stage & 3.937 & $2.025-7.654$ & $<0.001$ & & & ns \\
\hline Tumor size & 2.277 & $1.236-4.197$ & 0.008 & 1.955 & $1.002-3.811$ & 0.049 \\
\hline Residual tumor & 4.227 & $2.131-8.384$ & $<0.001$ & 3.784 & $1.858-7.707$ & $<0.001$ \\
\hline VEGFR-3 mRNA expression & 1.015 & $0.572-1.800$ & ns & & & ns \\
\hline VEGF-C mRNA expression & 1.768 & $0.982-3.183$ & 0.050 & & & ns \\
\hline VEGF-D mRNA expression & 0.845 & $0.445-1.604$ & ns & & & ns \\
\hline PDPN mRNA expression & 1.95 & $1.079-3.524$ & 0.027 & 2.081 & $1.121-3.865$ & 0.020 \\
\hline
\end{tabular}

HR, hazard ratio; CI, confidence interval; VEGF, vascular endothelial growth factor; VEGFR, VEGF receptor; PDPN, podoplanin; ns, not significant $(\mathrm{P}>0.05)$.

size, and depth of tumor invasion were associated with lymph node metastasis by performing univariate regression analysis. Multivariate logistic regression analysis also revealed PDPN mRNA expression, increasing tumor size and increasing depth of tumor invasion to be independent factors affecting lymph node metastasis. These findings indicate that PDPN, VEGF-C and VEGF-D mRNA expression were more significantly associated with lymphatic spread than hematogenous metastasis, highlighting their possible efficacy in predicting the nodal status of patients with esophageal cancer.

In the present study, a poor OS time was positively correlated with the overexpression of VEGF-C and PDPN in the esophageal cancer cells. Multivariate analysis identified tumor size, residual tumor and mRNA PDPN expression as independent factors of patient prognosis. Furthermore, the present study used the Kaplan-Meier method to determine that high VEGF-C expression was associated with a significantly shorter OS time compared with low VEGF-C expression. In agreement with this finding, Kitadai et al (25),
Kimura et al (26) and our previous study (8) used immunohistochemistry to demonstrate that the prognosis of patients with VEGF-C-positive tumors was significantly worse than that of patients with VEGF-C-negative tumors. Similarly, Okazawa et al (20) identified a significant difference in survival rates between groups with or without VEGF-C overexpression in patients with esophageal cancer. In addition, this study performed a multivariate analysis that determined that gender, age, VEGF-C expression and lymphatic invasion were all prognostic determinants in esophageal cancer. Using univariate survival analysis, Tanaka et al (21) determined a significant difference in OS between high and low VEGF-C mRNA expression in patients with esophageal cancer. Tong et al (22) performed immunohistochemical analysis and, using univariate and multivariate analysis, identified that overexpression of PDPN was both a prognostic factor and independent prognostic factor for 5-year disease-free survival. Furthermore, Rahadiani et al (23) demonstrated that PDPN overexpression was a prognostic factor for OS and 
disease-free survival, and an independent prognostic factor for disease-free survival.

The present results indicated that, as they are secreted by esophageal cancer cells, VEGF-C and PDPN may be able to induce and mediate tumor cell invasion, spread cancer cells beyond the primary tumor, and form a metastatic focus in the lymph nodes.

In conclusion, the current study identified that the expression of VEGF-C, VEGF-D and PDPN mRNA was significantly correlated with lymph node metastasis and tumor stage. In particular, PDPN overexpression was significantly associated with patients at a high risk of regional lymph node metastasis. Thus, VEGF-C and PDPN overexpression may be useful as possible indicators of poor prognosis, and PDPN overexpression may be applied as an independent prognostic marker in patients with esophageal cancer that have undergone potentially curative esophagectomy.

\section{Acknowledgements}

The present study was partially conducted within the Cancer/Mutagenesis research area of the Leading National Research Centre (KNOW). The study was supported by a grant obtained from the National Science Centre (no. N N403 282739).

\section{References}

1. Ferlay J, Shin HR, Bray F, Forman D, Mathers C and Parkin DM. Estimates of worldwide burden of cancer in 2008: GLOBOCAN 2008. Int J Cancer 127: 2893-2917, 2010.

2. Eloubeidi MA, Desmond R, Arguedas MR, Reed CE and Wilcox CM: Prognostic factors for the survival of patients with esophageal carcinoma in the US: The importance of tumor length and lymph node status. Cancer 95: 1434-1443, 2002.

3. Wilson M, Rosato EL, Chojnacki KA, Chervoneva I, Kairys JC, Cohn HE, Rosato FE Sr and Berger AC: Prognostic significance of lymph node metastases and ratio in esophageal cancer. J Surg Res 146: 11-15, 2008.

4. Veikkola T and Alitalo K: VEGFs, receptors and angiogenesis. Semin Cancer Biol 9: 211-220, 1999.

5. Achen MG and Stacker SA: Molecular control of lymphatic metastasis. Ann NY Acad Sci 1131: 225-234, 2008.

6. Stacker SA, Caesar C, Baldwin ME, Thornton GE, Williams RA, Prevo R, Jackson DG, Nishikawa S, Kubo H and Achen MG: VEGF-D promotes the metastatic spread of tumor cells via the lymphatics. Nat Med 7: 186-191, 2001.

7. Shida A, Fujioka S, Ishibashi Y, Kobayashi K, Nimura H, Mitsumori N, Suzuki Y, Kawakami M, Urashima M and Yanaga K: Prognostic significance of vascular endothelial growth factor D in gastric carcinoma. World J Surg 29: 1600-1607, 2005.

8. Kozlowski M, Naumnik W, Niklinski J, Milewski R, Dziegielewski P and Laudanski J: Vascular endothelial growth factor $\mathrm{C}$ and $\mathrm{D}$ exprossion correlates with lymph node metastasis and poor prognosis in patients with resected esophageal cancer. Neoplasma 58: 311-319, 2011.

9. Breiteneder-Geleff S, Soleiman A, Kowalski H, Horvat R, Amann G, Kriehuber E, Diem K, Weninger W, Tschachler E, Alitalo K, et al: Angiosarcomas express mixed endothelial phenotypes of blood and lymphatic capillaries: Podoplanin as a specific marker for lymphatic endothelium. Am J Pathol 154: 385-394, 1999
10. Kozłowski M, Naumnik W, Nikliński J, Milewski R, Lapuć G and Laudański J: Lymphatic vessel invasion detected by the endothelial lymphatic marker D2-40 (podoplanin) is predictive of regional lymph node status and an independent prognostic factor in patients with resected esophageal cancer. Folia Histochem Cytobiol 49: 90-97, 2011.

11. Kozłowski M, Niklińska W, Naumnik W, Laudański W, Milewski R and Nikliński J: Intratumoral lymphatic vessel density and intratumoral and peritumoral lymphatic vessel invasion as predictive factors of lymph node metastasis and prognostic factors in esophageal cancer. Kardiochir Torakochir Pol 10: 120-129, 2013.

12. Wicki A, Lehembre F, Wick N, Hantusch B, Kerjaschki D and Christofor G: Tumor invasion in the absence of epithelial-mesenchymal transition: Podoplanin-mediated remodeling of the actin cytoskeleton. Cancer Cell 9: 261-272, 2006.

13. Edge SB, Byrd DR, Compton CC, Fritz AG, Greene FL and Trotti A: Esophagus and esophagogastric junction. In: AJCC cancer staging manual. Edge SB, Byrd DR, Compton CC, Fritz AG, Greene FL and Trotti A (eds). 7th edition. Springer, New York, NY, pp103-107, 2010.

14. Schmittgen TD and Livak KJ: Analyzing real-time PCR data by the comparative C(T) method. Nat Protoc 3: 1101-1108, 2008

15. Sporn MB: The war on cancer. Lancet 347: 1377-1381, 1996.

16. Hirakawa S, Brown LF, Kodama S, Paavonen K, Alitalo K and Detmar M: VEGF-C-induced lymphangiogenesis in sentinel lymph nodes promotes tumor metastasis to distant sites. Blood 109: 1010-1017, 2007.

17. Kozłowski M, Laudański W, Mroczko B, Szmitkowski M, Milewski R and Łapuć G: Serum tissue inhibitor of metalloproteinase 1 (TIMP-1) and vascular endothelial growth factor A (VEGF-A) are associated with prognosis in esophageal cancer patients. Adv Med Sci 58: 227-234, 2013.

18. Nakayama Y, Matsumoto K, Nagato M, Inoue Y, Katsuki T, Minagawa N, Shibao K, Tsurudome Y, Hirata K, Higure A, et al: Significance of lymphangiogenesis as assessed by immunohistochemistry for podoplanin in patients with esophageal carcinoma. Anticancer Res 27: 619-625, 2007.

19. Kimura H, Kato H, Tanaka N, Inose T, Faried A, Sohda M, Nakajima M, Fukai Y, Miyazaki T, Masuda N, et al: Preoperative serum vascular endothelial growth factor-C (VEGF-C) levels predict recurrence in patients with esophageal cancer. Anticancer Res 28: 165-169, 2008.

20. Okazawa T, Yoshida T, Shirai Y, Shiraishi R, Harada T, Sakaida I, Abe T and Oka M: Expression of vascular endothelial growth factor $\mathrm{C}$ is a prognostic indicator in esophageal cancer. Hepatogastroenterology 55: 1503-1508, 2008.

21. Tanaka T, Ishiguro H, Kuwabara Y, Kimura M, Mitsui A, Katada T, Shiozaki M, Naganawa Y, Fujii Y and Takeyama H: Vascular endothelial growth factor C (VEGF-C) in esophageal cancer correlates with lymph node metastasis and poor patient prognosis. J Exp Clin Cancer Res 29: 83, 2010.

22. Tong L, Yuan S, Feng F and Zhang H: Role of podoplanin expression in esophageal squamous cell carcinoma: A retrospective study. Dis Esophagus 25: 72-80, 2012.

23. Rahadiani N, Ikeda JI, Makino T, Tian T, Qiu Y, Mamat S, Wang Y, Doki Y, Aozasa K and Morii E: Tumorigenic role of podoplanin in esophageal squamous-cell carcinoma. Ann Surg Oncol 17: 1311-1323, 2010.

24. Tzao C, Lee SC, Tung HJ, Hsu HS, Hsu WH, Sun GH, Yu CP, Jin JS and Cheng YL: Expression of hypoxia-inducible factor (HIF)-1alpha and vascular endothelial growth factor (VEGF)-D as outcome predictors in resected esophageal squamous cell carcinoma. Dis Markers 25: 141-148, 2008.

25. Kitadai Y,Amioka T,Haruma K, Tanaka S, Yoshihara M, Sumii K, Matsutani N, Yasui W and Chayama K: Clinicopathological significance of vascular endothelial growth factor (VEGF)-C in human esophageal squamous cell carcinomas. Int J Cancer 93: 662-666, 2001

26. Kimura Y, Watanabe M, Ohaga T, Saeki H, Kakeji Y, Baba H and Maehera Y: Vascular endothelial growth factor $\mathrm{C}$ expression correlates with lymphatic involvement and poor prognosis in patients with esophageal squamous cell carcinoma. Oncol Rep 10: 1747-1751, 2003. 